\title{
The impact of localized overlap eigenmodes on RMT measurements and topology
}

\author{
Anna Hasenfratz* \\ Department of Physics, University of Colorado, Boulder, CO-80309-390 \\ E-mail: anna@eotvos.colorado.edu
}

Roland Hoffmann

Department of Physics, University of Colorado, Boulder, CO-80309-390

E-mail: hoffmannepizero.colorado.edu

\section{Stefan Schaefer}

NIC, DESY, Platanenallee 6, D-15738 Zeuthen, Germany

E-mail: stefan.schaeferdesy.de

\begin{abstract}
The low energy eigenmodes of the continuum QCD Dirac operator are extended, but on the lattice, due to discretization effects, the Dirac operator can have localized eigenmodes. These nonphysical modes can introduce strong lattice artifacts for observables that are sensitive to chiral symmetry, especially in mixed action simulations. We study how these lattice artifacts depend on the parameters of the overlap operator and their effect on the distribution on the Dirac eigenmodes and the topological susceptibility.
\end{abstract}

The XXV International Symposium on Lattice Field Theory

July 30-4 August 2007

Regensburg, Germany

\footnotetext{
${ }^{*}$ Speaker.
} 


\section{Introduction}

Mixed action simulations combine the advantages of chiral operators in the measurement with relatively fast configuration generation, but their success to a large extent depends on how close the valence and sea quark actions are. At the very least one requires that the valence action does not introduce any new (large) lattice artifacts. Our goal is to find the "best" overlap action, in the sense of smallest lattice artifacts within a group of simple actions, to use with our ongoing Wilson action dynamical simulation[1-3]. In a mixed action situation the valence operator interacts with the sea quarks through the vacuum, therefore it is best to match the valence fermions to the dynamical ones via physical quantities that are sensitive to the vacuum but independent of the valence quark mass. We have considered two such observables, the topological susceptibility and the distribution of the infrared eigenmodes of the valence Dirac operator[4].

In the phase where chiral symmetry is spontaneously broken the low energy eigenmodes of the Dirac operator are expected to be extended, delocalized. The lattice Dirac operator can have many localized eigenmodes, but as long as these modes remain separate form the low energy infrared modes, they do not affect physical predictions. We have investigated the localization properties of the eigenmodes of the Wilson Dirac operator and several different overlap operators. We found that while both the Wilson and overlap operators have localized eigenmodes, these modes do not mix with the infrared modes of the Wilson Dirac operator, but can become part of the low energy spectrum of the overlap operator. The density of these non-physical modes depend on the parameters of the overlap construction, on the gauge configurations and on the lattice spacing. We can relate these modes to the localized modes of the kernel operator and argue that they are due to dislocations of the gauge configurations. These non-perturbative lattice artifacts can strongly affect chiral observables in mixed action simulations.

Our observation suggests that in order to minimize scaling violations in overlap simulations it is not sufficient to rely on perturbative $O(a)$ improvement but that non-perturbative lattice artifacts due to dislocations also have to be considered.

\section{Notations and parameters}

While this work is motivated by dynamical simulations with nHYP smeared improved Wilson fermions[1-3], the lattice artifacts of the overlap valence operators can be equally studied on quenched configurations. We used about $100012^{4}$ configurations generated with Wilson plaquette action at $\beta=5.8458(a \approx 0.12 \mathrm{fm})$.

Our definition of the massless overlap operator is

$$
D_{\mathrm{ov}}=R_{0}\left(1+d\left(d^{\dagger} d\right)^{-1 / 2}\right), \quad d=D_{K}-R_{0},
$$

where $D_{K}$ is the kernel operator and $R_{0}$ denotes the center of the overlap projection. We choose $D_{K}$ to be the Wilson operator with nHYP smeared gauge connections $[5,1]$, both unimproved and with tree level $\left(c_{\mathrm{SW}}=1\right)$ clover improvement.

The choice of the parameter $R_{0}$ in the overlap construction is rather arbitrary, as long as it is larger than the eigenvalues of the physical, infrared modes of the kernel operator but smaller than the doubler modes, and the resulting overlap operator is local. Since the infrared edge of 


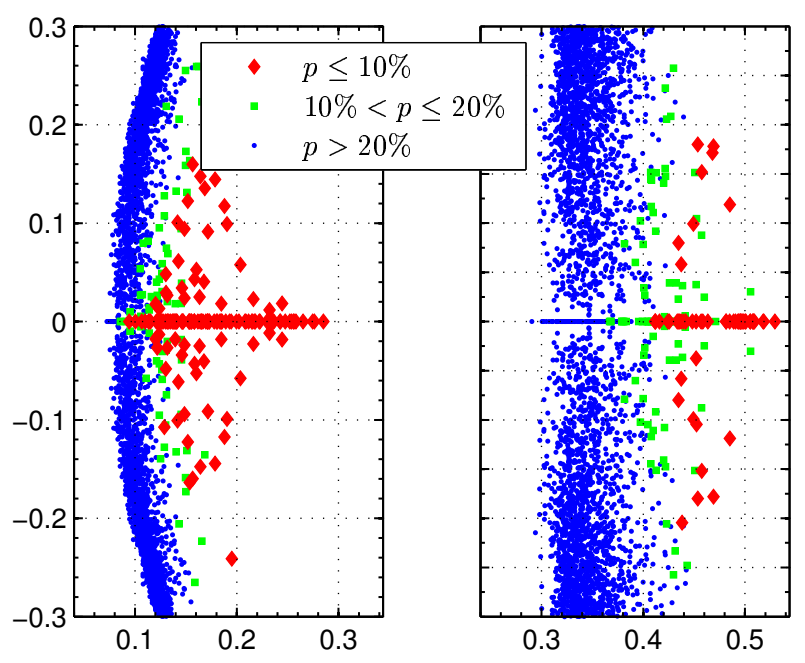

Figure 1: The spectrum of the two kernel operators used in this study. Both are nHYP smeared Wilson operators, one with tree level $c_{\mathrm{SW}}=1$ clover coefficient (left panel), the other with $c_{\mathrm{SW}}=0$ (right panel). The different plot symbols correspond to different localization levels of the corresponding eigenvectors .

the spectrum, $\lambda_{\text {crit }}$, varies with the kernel operator, the quantity $\Delta R_{0}=R_{0}-\lambda_{\text {crit }}$ characterizes the overlap operator better than $R_{0}$ itself. We have chosen two different $\Delta R_{0}$ values with both of our action, $R_{0}=1.0\left(\Delta R_{0} \approx 0.7\right)$ and $R_{0}=0.7\left(\Delta R_{0} \approx 0.4\right)$ with the unimproved $c_{\mathrm{SW}}=0$ action and $R_{0}=1.0\left(\Delta R_{0} \approx 0.92\right)$ and $R_{0}=0.3\left(\Delta R_{0} \approx 0.22\right)$ with the improved $c_{\mathrm{SW}}=1.0$ action. All four actions lead to a local overlap operator.

\section{The eigenvalue spectrum of the kernel and overlap Dirac operators}

First we consider the eigenvalue spectrum of the kernel operators. Figure 1 shows the 40 lowest magnitude eigenvalues on 100 configurations with both the $c_{\mathrm{SW}}=1$ and $c_{\mathrm{SW}}=0$ kernel actions. The spectrum of the nHYP smeared $c_{\mathrm{SW}}=1$ operator appears much more chiral than the unimproved one, its eigenvalues are concentrated around a unit circle. This is what makes this action appealing in dynamical simulations.

A simple and very intuitive measure of the localization of the eigenmodes is the participation number or inverse of the inverse participation ratio IPR [6]

$$
\begin{aligned}
p & =I P R^{-1} \\
I P R & =V \sum_{x}|\psi(x)|^{4},
\end{aligned}
$$

where $\psi(x)$ is the normalized eigenvector of the Dirac operator. In Figure 1 the different plotting symbols correspond to different participation numbers of the eigenmodes, and one observes a strong correlation between $p$ and $\Delta R$, the distance from the outer edge of the circle. Toward the center of the eigenvalue circle all modes appear to be localized with small $p$ for both actions. However the spectrum of the clover improved action has many more localized modes in the vicinity of the physical, IR range. 


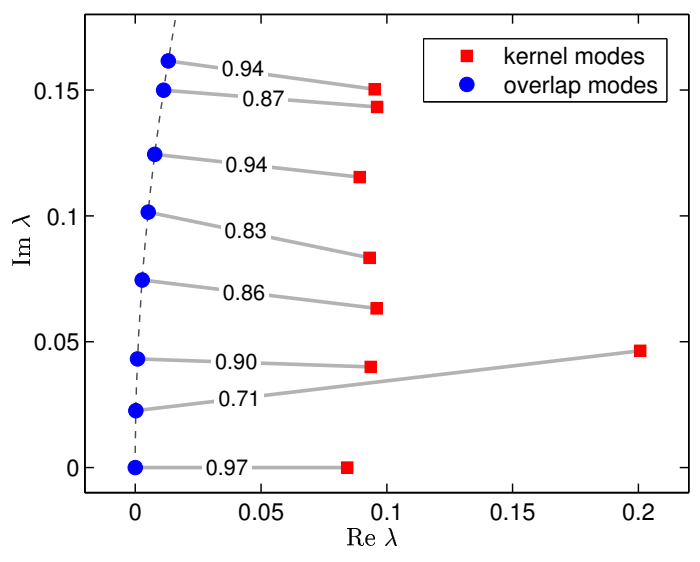

Figure 2: Low energy eigenvalues of the kernel and overlap operator on a configuration where the overlap operator has a localized IR mode. The lines connect the overlap and kernel modes with the highest overlap, and the magnitude of the inner product of the modes is also shown.

The participation number is only a qualitative measure: while a very small $p$ certainly implies a localized mode, a large value does not necessarily mean a coherent extended one. Finite volume analysis can distinguish the localized and extended modes. If the typical eigenmodes in a given region of the eigenvalue circle correspond to extended modes, their average participation $\bar{p}$ should be volume independent, while in the region where most of the eigenmodes are localized $\bar{p}$ will decrease with the inverse of the volume. Comparing data on $12^{4}$ and $16^{4}$ lattices we found constant $\bar{p}$ values for $\Delta R<0.03$ and $1 / V$ dependence for $\Delta R \geq 0.05$ for the $c_{S W}=1$ spectrum. This finite volume analysis suggests that on the $12^{4}$ lattices eigenmodes with participation number $p<0.40$ at $\Delta R \approx 0.05$ are already localized.

The overlap construction "projects" all the modes to the Ginsparg-Wilson circle. A typical example of how the overlap construction transforms the kernel modes is shown in Figure 2, where we compare the eigenvalues of the $c_{S W}=1$ kernel operator and the corresponding overlap operator. All but one of the kernel eigenmodes shown are extended with large participation numbers, the only exception is the mode in the inner part of the circle that has $p \approx 0.04$. The eigenmodes of the overlap operator are also extended with one exception, the mode with the lowest imaginary value has $p \approx 0.08$. The extended overlap eigenmodes connect strongly to a kernel mode, with overlap between the wave functions of $80 \%$ or larger as indicated in the figure. The extended, near infra-red eigenmodes change little under the overlap projection, their eigenvalues basically move straight out to the Ginsparg-Wilson circle. The localized mode, on the other hand, behaves differently. Both the overlap and kernel wave functions are sharply concentrated, they couple mainly to a small instanton or dislocation. The overlap of the wave functions is sizable, $\sim 70 \%$, but the eigenvalues are quite different. The overlap eigenvalue is small, the most infrared among the eigenmodes. In general localized modes tend to stay localized under the overlap projection, their overlap eigenvalue is frequently small, without modifying the eigenvalues of the extended modes.

To quantify the observations from Figures 1 and 2 we have measured the participation number of the low eigenmodes of our four overlap operators. Figure 3 shows the distribution for the first non-zero modes in the $v=1$ topological sector. The result supports what we have expected based 

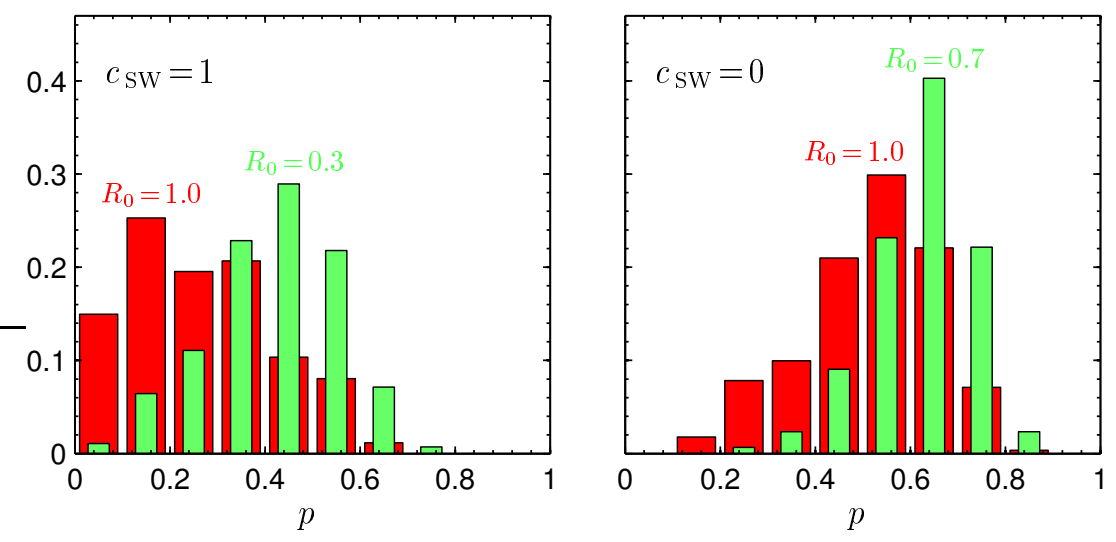

Figure 3: The distribution of the participation number of the first non-zero overlap eigenmodes in the $v=1$ sector, normalized by the number of configurations.

on the eigenmodes of the kernel operator. The $c_{\mathrm{SW}}=1$ improved action kernel operator with $R_{0}=1.0$ has a lot of localized modes - possibly more than $50 \%$ of the first eigenmodes are localized. The other actions are considerably better. When $R_{0}=0.3$ is used even with the clover improved action, many of the localized modes are already to the right of the overlap center and projected to the ultra-violet. Setting the clover coefficient $c_{\mathrm{SW}}=0$ has a similar effect. Even with $R_{0}=1.0$, corresponding to $\Delta R_{0}=0.70$, there are only a few localized modes, and their number drops even further when $R_{0}=0.7\left(\Delta R_{0}=0.40\right)$ is chosen.

\section{Consequences of localized overlap eigenmodes}

The distribution of the low lying Dirac eigenmodes should follow the universal predictions of Random Matrix Theory for the extended eigenmodes of the quenched systems if the volume is large enough, but localized modes embedded in the IR can spoil the agreement. According to RMT the probability distribution of a given eigenvalue of the Dirac operator in a fixed topological sector is a universal function and for quenched systems depends only on one free parameter, $\Sigma V / a$, where $\Sigma$ is the chiral condensate [7].

In Figure 4 we present our results for the cumulative (integrated) distribution using the four different Dirac operators and compare them to the RMT predictions. As is evident from Figure 4, the first eigenmodes are well described by RMT, but the agreement gets progressively worse for the higher modes. In general the $c_{\mathrm{SW}}=1.0$ operators are worse than the unimproved ones. While the $c_{\mathrm{SW}}=1.0$ smeared kernel action has much better chiral properties and the corresponding overlap operator is also more local than with the unimproved kernel, it also has many more localized eigenmodes in the IR overlap spectrum. These eigenmodes influence the distribution of the Dirac eigenmodes and ruin the agreement with the analytical predictions.

The topological susceptibility $\chi=\left\langle v^{2}\right\rangle / V$ is defined via the index of the overlap operator. It is identical to the sum of the chirality $( \pm 1)$ of the real modes of the kernel operator up to $\lambda<R_{0}$ [9]. The real modes of the kernel operator are easiest to identify by measuring the eigenvalues of the Hermitian operator $\gamma_{5} D_{K}$ and identifying when an eigenmodes crosses zero [10, 11]. In Figure 5 we show the dimensionless quantity $\chi r_{0}^{4}$ as a function of $R_{0}$ for the nHYP smeared $c_{\mathrm{SW}}=0$ and 1 

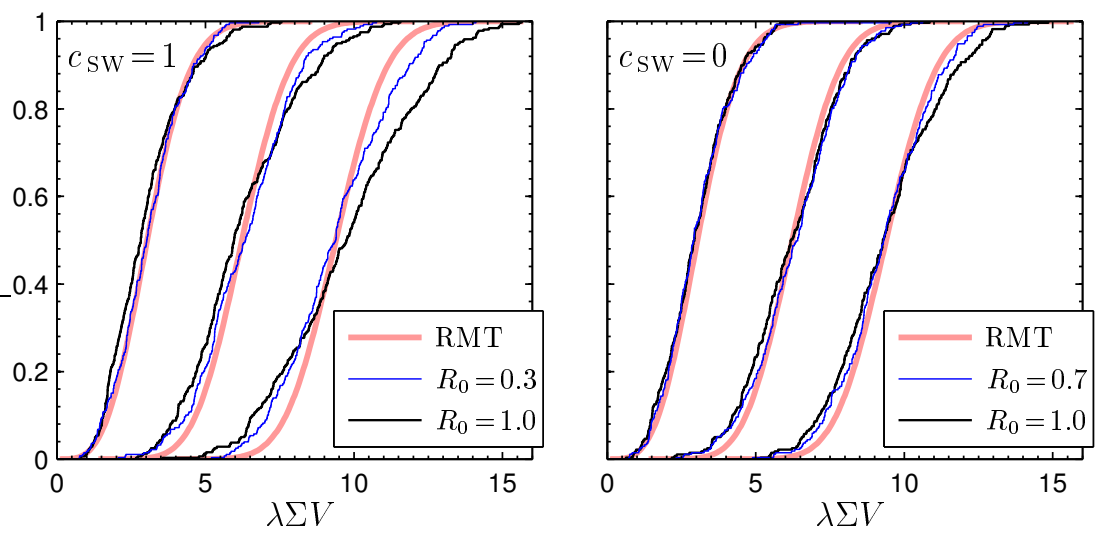

Figure 4: The cumulative distribution of the first three eigenmodes in the $v=1$ topological sectors. Left panel: $c_{\mathrm{SW}}=1$; right panel: $c_{\mathrm{SW}}=0$. The smooth thick lines are the RMT predictions.

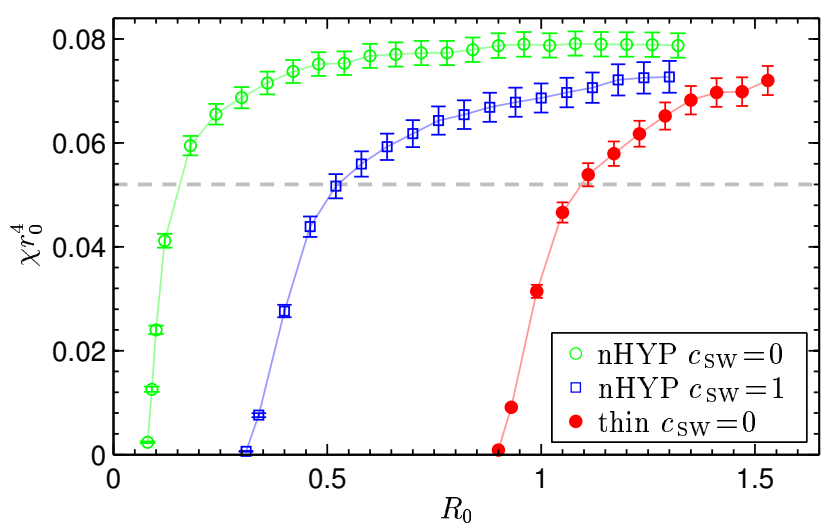

Figure 5: The topological susceptibility as a function of the overlap parameter $R_{0}$ with thin link and nHYP smeared overlap. The dashed horizontal line is the continuum prediction from Ref. [8].

kernel overlap actions, and also for the unimproved thin link kernel overlap action. To set the scale we use $r_{0} / a=4.032$ from Ref. [12]. Recent studies of the topological susceptibility using a pure gauge $F \tilde{F}$ topological charge operator predict $\chi r_{0}^{4}=0.0524(13)$ [8], while calculations with a thin link overlap operator give $\chi r_{0}^{4}=0.059(3)$ [13] in the continuum limit. In Figure 5 we observe not only large cut-off effects, but strong dependence on the $R_{0}$ parameter, especially for the $c_{\mathrm{SW}}=0$ actions. This is the consequence of the large number of real eigenmodes toward the center of the eigenvalue circle seen in Figure 1. Most of these modes are lattice artifacts, dislocations. Overlap operators with smaller $R_{0}$ values are less sensitive to these inner modes and therefore show smaller lattice artifacts.

Comparing results we obtained for the topological susceptibility and for the eigenvalue distributions we observe that lattice artifacts, or deviation form the continuum, correlate closely for both observables with the density of localized overlap eigenmodes (Fig. 3). 


\section{Conclusion and Discussion}

We have investigated the lattice artifacts of different overlap operators in quenched systems as reflected by the topological susceptibility and the distribution of the low energy eigenmodes. We related the observed cut-off effects to the existence of localized low energy eigenmodes in the overlap spectra.

While these localized modes, due to lattice dislocations, are lattice artifacts and will go away in the continuum limit, their presence can cause significant scaling violations at finite lattice spacing. One can minimize these by choosing a better kernel operator, like the nHYP smeared operator we considered here, and by tuning the $R_{0}$ parameter of the overlap construction as small as the locality of the overlap operator would allow.

In this paper we considered only quenched systems, but mixed action simulations suffer from the same problem. Fully dynamical overlap simulations should fare better as there the localized eigenmodes are suppressed just like any other small eigenvalue mode, so while they are present, their number is at least not inflated. Nevertheless an overlap operator that has small lattice artifacts in quenched should also have smaller lattice artifacts in dynamical simulations.

\section{Acknowledgment}

This work was partially supported by the US Department of Energy.

\section{References}

[1] A. Hasenfratz, R. Hoffmann and S. Schaefer, JHEP 05 (2007) 029 [hep-lat / 0702028 ].

[2] A. Hasenfratz, R. Hoffmann and S. Schaefer, arXiv:0709.4130 [hep-lat].

[3] A. Hasenfratz, R. Hoffmann and S. Schaefer PoS (LATTICE 2007) 104.

[4] A. Hasenfratz, R. Hoffmann and S. Schaefer, arXiv:0709.0932 [hep-lat].

[5] A. Hasenfratz and F. Knechtli, Phys. Rev. D64 (2001) 034504 [hep-lat/ 0103029 ].

[6] C. Gattringer, M. Göckeler, P. E. L. Rakow, S. Schaefer and A. Schäfer, Nucl. Phys. B617 (2001) 101-116 [hep-lat/0107016].

[7] P. H. Damgaard and S. M. Nishigaki, Phys. Rev. D63 (2001) 045012 [hep-th/ 0006111 ].

[8] S. Durr, Z. Fodor, C. Hoelbling and T. Kurth, JHEP 04 (2007) 055 [hep-lat/ 0612021 ].

[9] F. Niedermayer, Nucl. Phys. Proc. Suppl. 73 (1999) 105-119 [hep-lat/ 9810026 ].

[10] R. G. Edwards, U. M. Heller and R. Narayanan, Phys. Rev. D59 (1999) 094510 [hep-lat/9811030].

[11] L. Del Debbio and C. Pica, JHEP 02 (2004) 003 [hep-lat/ 0309145$].$

[12] S. Necco and R. Sommer, Nucl. Phys. B622 (2002) 328-346 [hep-lat/ 0108008 ].

[13] L. Del Debbio, L. Giusti and C. Pica, Phys. Rev. Lett. 94 (2005) 032003 [hep-th/ 0407052 ]. 